\title{
Bi-functional Alkylating Agent VAL-083
}

National Cancer Institute

\section{Source}

National Cancer Institute. Bi-functional Alkylating Agent VAL-083. NCI Thesaurus. Code C99224.

A bi-functional alkylating agent, with potential antineoplastic activity. Upon administration, VAL-083 crosses the blood brain barrier (BBB) and appears to be selective for tumor cells. This agent alkylates and crosslinks DNA which ultimately leads to a reduction in cancer cell proliferation. In addition, VAL-083 does not show cross-resistance to other conventional chemotherapeutic agents and has a long half-life in the brain. 